
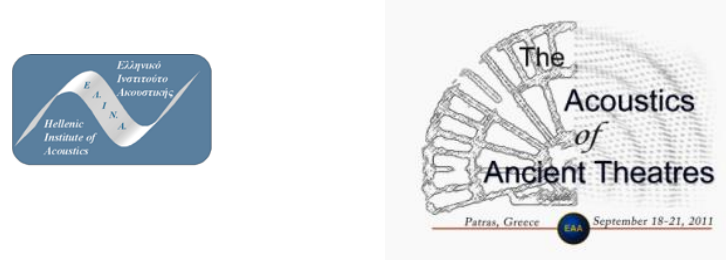

The Acoustics of Ancient Theatres Conference

Patras, September 18-21, 2011

\title{
RECREATING THE SOUND OF STONEHENGE
}

\author{
Bruno Fazenda (1), Ian Drumm (1)
}

1) Acoustics Research Centre, The University of Salford, Salford, UK

e-mail: b.m.fazenda@salford.ac.uk

\begin{abstract}
Stonehenge is the largest and most complex ancient stone circle known to mankind. In its original form, the concentric shape of stone rings would have surrounded an individual both visually and aurally. It is an outdoor space and most archaeological evidence suggests it did not have a roof. However, its large, semi-enclosed structure, with many reflecting surfaces, would have reflected and diffracted sound within the space creating an unusual acoustic field for the Neolithic Man.

This project has reconstructed the acoustic sound field of Stonehenge based on measurements taken at a full size replica in Maryhill, USA. Acoustic measurements were carried out using modern techniques and the response collected in both mono and B-Format at various source-receiver positions within the space. A brief overview of a few acoustic parameters together with a comparison to a recent measurement in the current Stonehenge site is provided.

The auralisation process presented uses a hybrid ambisonic and wave field synthesis (WFS) system. Anechoic recordings were rendered as focussed sources in the WFS system whilst their reverberant B-Format counterpart was rendered using ambisonic technology. Using this technology, the original acoustics of the space have been reconstructed for listeners.

The paper discusses the measurement, acoustic response and rendering of single, focused sources within the space. The use of multiple B-Format impulse responses for various source positions allows embedding of these focussed sources within the reverberant field.
\end{abstract}

Keywords

Archaeoacoustics; auralization; wave field synthesis; ambisonics

\section{Introduction}

The preservation and recreation of the acoustic characteristics of ancient spaces serves various important purposes: It is vital in the reconstruction of theatres [1]; aids in the archaeological interpretation of important buildings and heritage sites, some of which may not exist in their original form [2,3]; allows experiencing of their soundfields through auralisation which has benefits for public interactive applications as well as in more ecologically valid archaeological studies. 
The work presented here aims to report on the recreation of the acoustic sound field of Stonehenge, a well known pre-historic stone circle situated in Wiltshire, England. Due to its circular shape and the large number of enclosing surfaces, a listener inside the original stone circle would be presented with an unusual sound field for an outdoor space. However, as it currently stands, the monument is in ruins and only a few of the original stones still remain standing. As such, measuring and experiencing the acoustic sound field of Stonehenge in its final construction phase (ca. 1500 B.C.) has hitherto not been possible.

A full size identical replica of the Stonehenge monument exists on the banks of the Columbia river in Maryhill, Washington, USA. The stone positions and sizes have been replicated using concrete, the surfaces of which have been worked in an attempt to simulate stone roughness. The 'stones' in the Maryhill site are, in general, more regular and do not replicate those at Stonehenge in absolute detail (see Figure 1). It may however be argued that some of the more general acoustic effects, such as reflection patterns and reverberation characteristic of Stonehenge, may also be found at Maryhill.

A series of acoustic measurements based on B-Format impulse responses has been carried out at the Maryhill site in order to obtain data for auralisation of the space using a hybrid approach that combines wave field synthesis and ambisonic reproduction.

This paper describes the measurement and auralisation process and is organised as follows: Section 2 gives a brief overview of Stonehenge including brief results of acoustic measurements taken within the stones; Section 3 discusses measurement and results for the acoustic response of the Maryhill site; Section 4 discusses the rendering methods for focused sources through the wave field synthesis system and the manipulation of the B-Format impulse responses for auralisation of the space; a summary is presented in section 5.
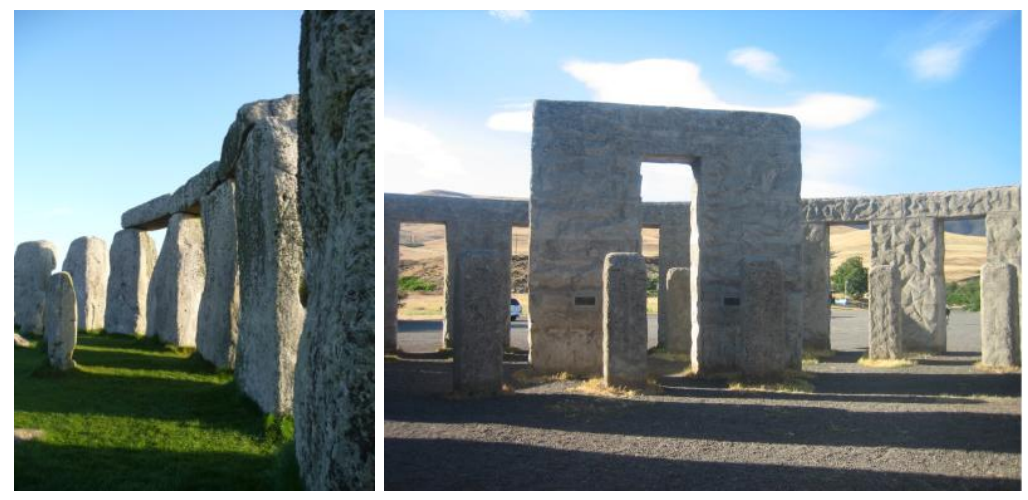

Figure 1 - Photos of Stonehenge (left) and Maryhill (right) sites

\section{Stonehenge}

The Stonehenge monument is characterized by concentric stone rings of various sizes (Figure 2). The outer circle is composed of 30 upright sarsen stones, 17 of which are still standing (shaded in Figure 2). The tops of the upright sarsen stones were original linked by an horizontal lintel ring. Inside the sarsen ring there was a $2^{\text {nd }}$ circle of smaller blue stones. A horse shoe shape of blue stones and the 5 large trilithons form the $3^{\text {rd }}$ inner semi-enclosure. More detailed information about the site can be found at [4].

Acoustic measurements have recently been carried out inside the Stonehenge circle using the balloon burst method [5] to obtain its impulse response (IR). Energy-Time 
Curves have been determined from measured IRs as follows. Given a time domain impulse response $h(t)$, the linear scale envelope time curve can be obtained from:

$$
e(t)=|h(t)+j \hat{h}(t)|
$$

Where $\widehat{h}(t)$ is the Hilbert transform of the signal $h(t)$ given by

$$
\hat{h}(t)=\frac{1}{\pi} \int_{-\infty}^{\infty} \frac{h(\tau) d \tau}{t-\tau}
$$

Assuming $h(t)$ denotes acoustic pressure, the Heyser energy time curve in decibel scale normalised to the direct source, can be obtained from

$$
\operatorname{ETC}(t)=20 \log \left[\frac{h(t)}{h_{\text {direct }}}\right]
$$

The ETC obtained for source and receiver in the centre of Stonehenge (indicated by the star symbol in Figure 2) is shown in Figure 3.

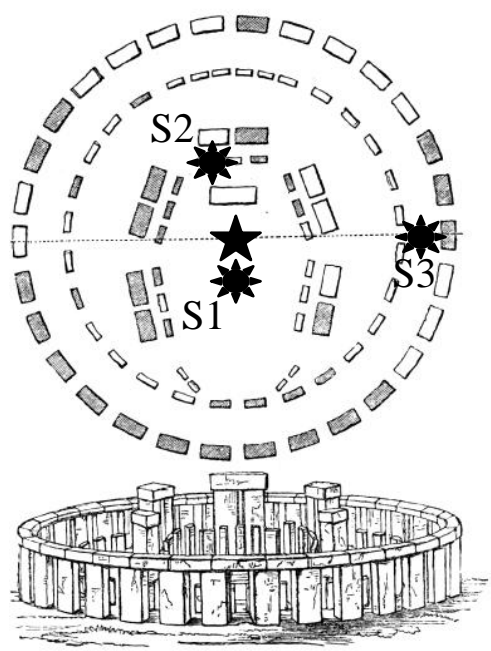

Figure 2- Diagram of Stonehenge circle. Receiver position is marked with a 5 point star. Source positions used for the B-Format measurements are indicated in 8 point stars.

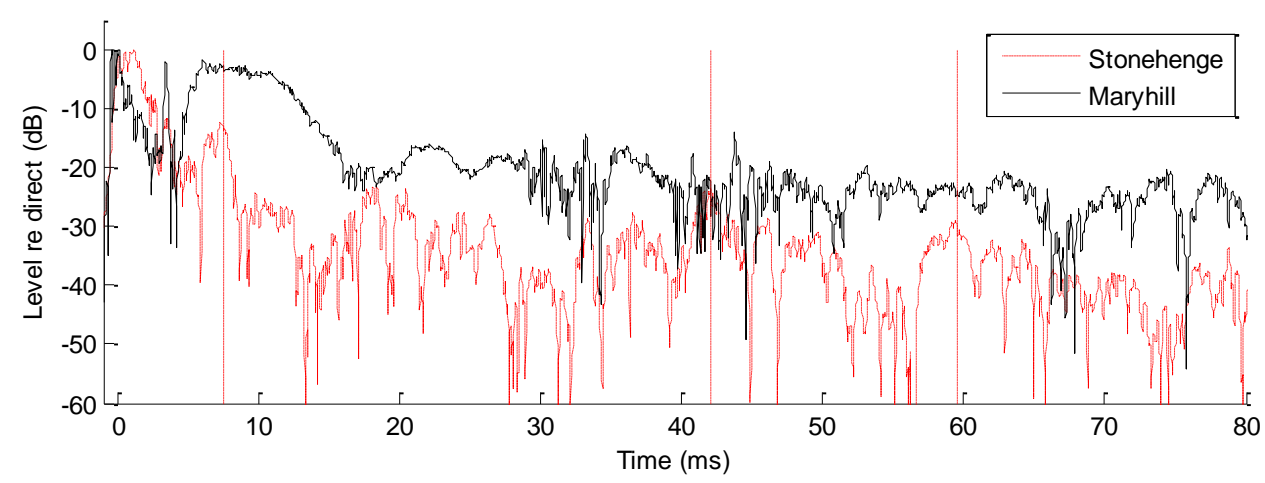

Figure 3- ETCs derived from single channel impulse responses. Stonehenge (red) and Maryhill (black) are provided. The vertical lines at $7.5 \mathrm{~ms}, 42 \mathrm{~ms}$ and $60 \mathrm{~ms}$ indicate specular reflections obtained in the Stonehenge measurement 
The ETC response measured in Stonehenge shows specular reflections at at $7.5 \mathrm{~ms}$, $42 \mathrm{~ms}$ and $60 \mathrm{~ms}$, although depending on which subjective data is used [eg: 6,7], they barely rise above the reflection detection threshold. This reflected energy originates from the floor, the nearer toppled stones and trilithons and the stones further away in the outer sarsen circle. Indeed, anecdotal evidence from standing inside the stone circle revealed a faint slap-back type echo arising mainly from the intact portion of the outer sarsen ring where the longer time of arrival (> $40 \mathrm{~ms}$ ) allows the definition of a discernible reflection.

The reverberation times for this measurement have been determined according to ISO3382. However, because the balloon burst method provides a very low signal to noise ration, the portion of decay curve used to extrapolate RT has been truncated between $-5 \mathrm{~dB}$ and $-15 \mathrm{~dB}$ for frequencies below $200 \mathrm{~Hz}$ and between $-5 \mathrm{~dB}$ and $-25 \mathrm{~dB}$ for frequencies above. Results are indicated in Table 1. As expected from an outdoor site, the RT values are quite low, averaging 0.33 seconds in the $63 \mathrm{~Hz}$ to $8000 \mathrm{~Hz}$ range.

These results confirm the expected notion that Stonehenge, in its current form, has few noteworthy acoustic features. Notwithstanding, in its final stage circa 1500 B.C., it is likely that the sound field within Stonehenge would have revealed a different character. Indeed, one could argue that the large number of enclosing stones, of different sizes and shapes and at different distances, would have created significant levels of diffusion and specular reflections should as those measured in the current monument would have been broken by multiple reflection paths arriving in succession at a listener position. It is also likely that, enclosed by many reflective surfaces, the space would have sustained a significant level of reverberation that might have been unusual for people visiting the site.

Table 1 - Octave band reverberation times calculated for Stonehenge and Maryhill sites. ' $r$ ' indicates the correlation coefficient for extracting the slope from the Schroeder integration.

\begin{tabular}{|r|c|c|c|c|}
\cline { 2 - 5 } \multicolumn{1}{c|}{} & \multicolumn{2}{c|}{ Stonehenge } & \multicolumn{2}{c|}{ Maryhill } \\
\hline $\mathbf{f c}(\mathbf{H z})$ & $\mathbf{R T}(\mathbf{s})$ & \multicolumn{1}{c|}{$\mathbf{r}$} & $\mathbf{R T}(\mathbf{s})$ & $\mathbf{r}$ \\
\hline 63 & 0.45 & -0.99 & 1.18 & -0.98 \\
\hline 125 & 0.11 & -1.00 & 0.98 & -0.86 \\
\hline 250 & 0.39 & -0.97 & 0.70 & -0.99 \\
\hline 500 & 0.17 & -0.95 & 0.50 & -0.96 \\
\hline 1000 & 0.32 & -0.95 & 0.52 & -0.97 \\
\hline 2000 & 0.36 & -0.98 & 0.47 & -0.99 \\
\hline 4000 & 0.47 & -0.95 & 0.33 & -0.97 \\
\hline 8000 & 0.33 & -0.96 & 0.30 & -0.98 \\
\hline Avg & 0.33 & & 0.62 & \\
\hline
\end{tabular}

\section{Acoustic measurement of Maryhill}

Given Stonehenge is in ruins, a faithful model of the site is required to obtain reliable acoustic measurements that may provide an indication of the acoustic characteristics of such a building. Such model exists in Maryhill, Washington state, USA. The Maryhill Stonehenge is a full size, concrete replica of Stonehenge built in 1929 as a 
memorial to the soldiers of the First World War. The acoustic measurements taken at the Maryhill site and used here for an auralisation of the space are currently the closest representation of the acoustic sound field present during the last phase of Stonehenge construction.

Techniques for measuring the acoustic characteristics of performance spaces have been widely reported [8,9]. ISO3382 sets the standard for such measurements although it does not acknowledge or utilize the advantages brought about by the use of pressureparticle velocity probes such as the Soundfield microphone which is now becoming ubiquitous in measurement-to-auralisation work such as the one presented here. The use of Soundfield microphones for capturing spatial sound properties of a space and the resulting B-Format output for rendering through varied electroacoustic formats (binaural, stereo, 5.1, ambisonic and wavefield synthesis) is also becoming more common and the reader is referred to the following references for further detail $[10,11,2 ; 12]$.

The measurement method used in the present work follows standard procedure established in the work referenced above. In particular, a dodecahedronic source was paired with a sub-woofer to adequately cover the audio frequency range between $40 \mathrm{~Hz}$ and $10 \mathrm{kHz}$ with an omnidirectional polar response. The excitation signal used was a 10 second log-sweep. The acoustic response of the space was captured using both an omnidirectional reference microphone (to obtain conventional impulse response and derive standard acoustic measures as prescribed in ISO3382) and a soundfield ST250 to capture 4 coincident impulse responses containing one omnidirectional pressure response and 3 orthogonal directional responses from which directional information and spatial auralisation data can be derived. The subwoofer was placed on the floor and the dodecahedron was placed directly above it at a height of approximately $1.6 \mathrm{~m}$. Microphones were positioned at an average head height of $1.6 \mathrm{~m}$. All measurements presented for the Maryhill site were obtained using WinMLS software, a modern personal computer and a Focusrite Saffire Pro 10 soundcard. As measurements were taken outdoor, power was provided via a combustion generator where due care was taken to prevent noise contamination onto measured responses. Similarly, and due to proximity of roads, environmental conditions and public access to the site, every measurement was checked for adequate signal to noise ratio to ensure validity of data.

The ETC for a source placed in the centre of the space and obtained with a single channel reference measurement also in the centre is shown in Figure 3 (solid line). In comparison with the measurement taken at Stonehenge in identical source-receiver relationship (Figure 3 red line), it is clear that the Maryhill monument exhibits significant reflected energy. Interestingly, there are no defined specular reflections suggesting the space might support something closer to a diffuse field. Reverberation times for the Maryhill site are indicated in Table 1. As expected, the values for RT are significantly higher than those measured at Stonehenge since, at least in the horizontal axis, the larger number of surrounding stone surfaces offer a more enclosed environment. This effect is clearly perceived as one walks into the space and the auralisation this work refers to also conveys these effects quite clearly.

For the purposes of auralising the space, measurements were taken at a single receiver position, placed at the centre of the stone circle as indicated (by the star symbol) in Figure 2.

- Three different source positions were used to generate various acoustic responses. These are also indicated in Figure 2 (using 'S\#'). 
The B-Format impulse response measured in the centre of the Maryhill monument is shown in Figure 4.
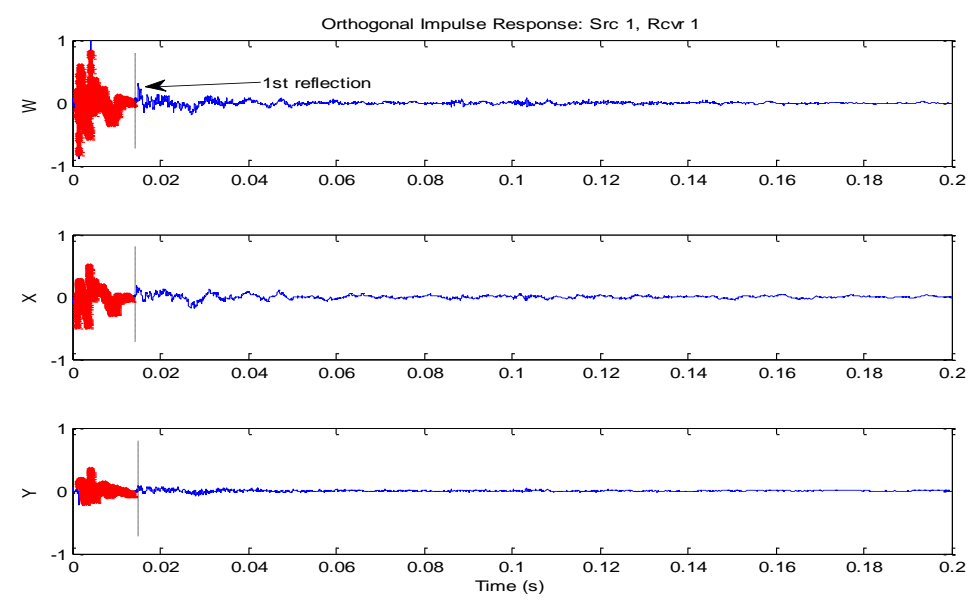

Figure 4 - Orthogonal Impulse Responses measured in Maryhill (a full size identical replica of Stonehenge). Only W,X and Y signals are represented

\section{Rendering System}

The aim of the system is to render sound sources and their reverberation as would be experienced within the original Stonehenge. The method developed is simple yet novel:

- 'Dry' sound sources are rendered in the space as focussed sources using wave field synthesis principles. The system allows real time movement of these sources.

- The 'acoustic response' of the space is obtained through convolution of the dry sources and an edited version of the B-Format impulse responses obtained within it. These IRs no longer contain the 'direct sound' information.

A listener in the rendering system is thus subjected to the correct directional cues generated from the reconstructed wavefronts for the focused sources and the ecologically valid environmental reflections of the space.

The rendering system used here has been fully developed within the Acoustics Research Centre at the University of Salford and is based on commercially available hardware comprised of 64 audio channels to drive an octagon shaped array of 64 loudspeakers. The wavefield synthesis and ambisonic algorithms and control is based on our own developed $\mathrm{C}++/ \mathrm{C \#} / \mathrm{WASAPI}$ software application.

The WFS part of the system may recreate the wavefronts of multiple arbitrarily positioned virtual sources both outside and within the confines of the speaker array thus emulating the distribution of sound waves in a volume as would be experienced by a user at any given position within that volume. As such, the WFS system it doesn't tie users to a sweet spot.

The ambisonic sources are rendered simultaneously through the same system. However, due to its underlying theory and its first order limitation in this case, the ambisonic cues are only correct to a centrally placed listener. 


\subsection{Focused sources in WFS}

Wavefield synthesis employs the Huygen's Principle which essentially states that wavefronts can be created by the cumulative effect of secondary sources just behind them. Given a plane of monopole sources the pressure at the receiver point in the volume can be predicted by adding secondary source contributions using a 'half space' simplification of the Kirchhoff-Helmholtz integral. Therefore, in theory, desired wavefields within a volume can be constructed from a set of enclosing surfaces of secondary sources.

Considering a virtual source located in space such that its position vector is $r_{s}$, the pressure at $r_{v}$ as function of frequency $\omega$ can be considered as a sum of contributions of a driving function $D$ at secondary source (i.e. loudspeaker) positions $r_{l}$ such that

$$
p_{r}(\omega) \propto \int_{S} D \frac{e^{-j k\left|\mathbf{r}_{v}-\mathbf{r}_{1}\right|}}{\left|\mathbf{r}_{v}-\mathbf{r}_{1}\right|} d S
$$

Typically the driving function includes a window $a$ to ensure only forward propagating waves reach the listener and a stationary phase approximation to represent vertical contributions at a given loudspeaker since linear arrays (not planes) are used. The term $S$ represents the sound signal to be rendered, $n$ the direction vector of the loudspeaker and $s=j \omega$.

$$
D=-2 a \sqrt{2 \pi\left|\mathbf{r}_{l}-\mathbf{r}_{r e f}\right|}\left(\frac{\left(\mathbf{r}_{l}-\mathbf{r}_{s}\right)^{T} \mathbf{n}}{\left(\mathbf{r}_{l}-\mathbf{r}_{s}\right)^{2}}\right) \frac{1}{\sqrt{c}}\left(s+\frac{c}{\left|\left(\mathbf{r}_{l}-\mathbf{r}_{s}\right)\right|}\right) \frac{1}{s} e^{-j k\left|\left(\mathbf{r}_{l}-\mathbf{r}_{s}\right)\right|} S(\omega)(5)
$$

In practice virtual sources are positioned within the world space coordinate system and their contributions to the respective driving functions are evaluated in the time domain, hence rendered by setting amplitudes, delays and coefficients of an IIR filter (which is used to account for the source spectra being a function of $r_{s}$ ).

\subsection{Room acoustic responses in ambisonic}

The dry mono focussed sources are 'placed' within Stonehenge by convolving them with the B-Format IRs thus providing a spherical harmonic decomposition of the sources in the reverberant sound field. However, in order to correctly reproduce only the early reflections and reverberation of the space, the direct sound and first reflection must be identified in the impulse responses.

The identification of the onset on the impulse responses was performed using a technique based on measuring the variation of temporal energy contained in the impulse response and finding where the ratio of consecutive analysis windows is maximum [13]. The impulse response is then trimmed to the index corresponding to its onset. The reflection onset times (ROT) has been obtained through a running kurtosis method as established in [14]. $\mathrm{ROT}_{1}$ is then used to determine the point before which the impulse response is padded with zeros to remove the direct sound but maintain the correct propagation length between source and receiver. 
Rendition of the environmental 'acoustic response' contained in B-Format is then obtained by applying Gerzon's equations [15] across all loudspeakers with relative contributions of $\mathrm{X}$ and $\mathrm{Y}$ being functions of the angle between $r_{l}-r_{s}$ and $n$.

For a centrally placed listener heading in any direction, the rendered source cues (directional and amplitude) are correct for sources placed at the positions where the BFormat IRs were measured. If real time movement of the focused sources is required or the listener moves within the space away from the central position, the early reflection cues will no longer be physically correct. A reasonable assumption is made that the reverberant cues will not make a significant contribution to subjective source localisation, and indeed this was the case at a few event demonstrations of the system, particularly if the focussed source cues are correctly maintained. Ideally, in the case of moving sources or listener, interpolating between a comprehensive grid of source-receiver IRs obtained on site would give a more objectively accurate representation of the reflective profile of the space but at an increased operational cost.

\subsection{Positioning of sources in the rendering space}

A folk ensemble comprised of two singers, one percussion instrument and a choir was rendered within the loudspeaker system. Source positions marked 2 and 3 in Figure 2 were selected for rendering since the B-Format IRs obtained at these positions contain the physically correct spatial cues. Given the scarce number of B-Format source positions, an extra 2 source positions were generated within the rendering space by inverting the velocity sensitive channels of the corresponding IRs prior to convolution. I.e. 2 further source positions $-\mathrm{S} 2$ and $-\mathrm{S} 3$, diametrically opposite to their relevant counterparts, were generated by inverting the corresponding $\mathrm{Y}$ and $\mathrm{X}$ signals of the corresponding BFormat IRs. The authors are aware that this latter technique will not represent the acoustic environment accurately but rather flip it in terms of side to side or front to back. It is nevertheless a complementary tool that may be used to obtain more source positions when only a few measurements are available without introducing gross inaccuracies. Furthermore, editing the temporal and amplitude response of reflections in the obtained IRs allows further manipulation of the resulting directional cues and reverberation characteristics. Once again, such techniques, as are already available in convolution reverb applications [16], will not be entirely accurate as they distort the physical characteristics of the space but are available to add flexibility to the method. The perceptual benefits of such techniques when compared to the more costly option of comprehensive B-Format IR measurement grids and associated interpolations is an issue for further investigation.

\section{Summary}

A brief overview of acoustic measurements taken at Stonehenge and at a full size identical and complete replica of it at Maryhill, USA, has been reported. It has been shown that although the acoustic sound field currently present at the Stonehenge monument reveals a few weak specular reflections and weak acoustic reverberation, its original form might have presented a much more interesting sound field with significant diffusion properties and a noticeable reverberation.

The work presented here reported on the recreation and auralisation of this sound field through a novel hybrid system based on wavefield synthesis and ambisonic render- 
ing techniques. It has been shown that, although there are still a few limitations, in particular when the feature of moving sources is used, the proposed system is able to provide a very realistic and ecologically valid rendition of the measured spaces.

The rendering system has been demonstrated at recent public and private events and may be experienced at the Acoustics Research Centre at the University of Salford. Rendition examples using more conventional methods may be listened to at [17].

Future work on this project is now focussed on the correct manipulation of BFormat IRs for moving sources and on the use of finite difference time domain models to allow rendition of sources placed anywhere in the space.

\section{Acknowledgement}

We thank the Schools of Computing and Engineering and Music, Humanities and Media at the University of Huddersfield for supporting the measurement trips; the Maryhill Museum of Art for access to the Maryhill site; and English Heritage for providing access to the Stonehenge site.

\section{References}

[1] Farina, Angelo; Tronchin, Lamberto (2004). Advanced techniques for measuring and reproducing spatial sound properties of auditoria. Proc. of International Symposium on Room Acoustics Design and Science (RADS), 11-13 April 2004, Kyoto, Japan.

[2] D.T Murphy, "Archaeological Acoustic Space measurement for convolution reverberation and auralization applications", Proc. of the 9th Int. Conference on Digital Audio Effects (DAFX'06), Montreal, Canada, September 18-20, 2006.

[3] David Lubman "Acoustical solutions to archaeological mysteries at Chichen Itza's temple of Kukulkan.” (A) , J. Acoust. Soc. Am. 128, 2329 (2010)

[4] http://www.stonehenge.co.uk/

[5] Jukka Pätynen, Brian F.G. Katz, and Tapio Lokki, "Investigations on the balloon as an impulse source”, J. Acoust. Soc. Am. 129, EL27 (2011)

[6] S.E. Olive and F.E. Toole, "The Detection of Reflections in Typical Rooms", J. Audio Eng, Soc., vol. 37, pp. 539-553 (1989 July/August)

[7] Haas, H. "The Influence of a Single Echo on the Audibility of Speech", JAES Volume 20 Issue 2 pp. 146-159; March 1972

[8] Pompoli, Roberto; Prodi, Nicola "Guidelines for Acoustical Measurements inside Historical Opera Houses: Procedures and Validation". Journal of Sound and Vibration232 (1): 281-301. (April 2000).

[9] Farina, A., Ayalon, R., "Recording Concert Hall Acoustics for Posterity", AES 24th International Conference on Multichannel Audio, Banff, Canada, June 26-28 2003

[10] D. T. Murphy, "Multi-channel Impulse Response Measurement, Analysis and Rendering in Archaeological Acoustics", 119th AES Convention, Paper No. 6532, New York, USA, Oct 7-10, 2005

[11] Merimaa, Juha; Pulkki, Ville, "Spatial Impulse Response Rendering I: Analysis and Synthesis", JAES Volume 53 Issue 12 pp. 1115-1127; December 2005

[12] A. Farina and L. Tronchin, "Advanced techniques for measuring and reproducing spatial sound properties of auditoria", International Symposium on Room Acoustics: Design and Science 2004, Kyoto, Japan, April 11-13 2004. 
[13] G. Defrance, L. Daudet, and J.-D. Polack, "Finding the onset of a room impulse response: Straightforward?," J.Acoust. Soc. Am. 124, EL248-EL254 (2008).

[14] Usher, J., "An improved method to determine the onset timings of reflections in an acoustic impulse response", EL172, J. Acoust. Soc. Am., 127, 4, April 2010

[15] Gerzon, M., "Periphony: With-height sound reproduction", JAES Jan/Feb. 1973, Vol.21, No:1

[16] Ben-Hador, R. \& Neoran, I., "Capturing Manipulation and Reproduction of Sampled Acoustic Responses"; $117^{\text {th }}$ AES Convention, San-Francisco, (2004)

[17] http://www.acoustics.salford.ac.uk/res/fazenda/acoustics-of-stonehenge/ 